\title{
Determinando Presas: Primeros Resultados Osteométricos para la Identificación de Especies de Otáridos en Concheros de Norpatagonia (Río Negro, Argentina)
}

\author{
Determining Preys: First Results of Osteometric Identification of Otariids from \\ Northern Patagonia Shellmiddens (Rio Negro, Argentina)
}

Florencia Borella ${ }^{\mathrm{i}} \mathrm{G}$. Lorena L'Heureux ${ }^{\mathrm{ii}}$

RESUMEN

Análisis arqueofaunísticos realizados en la costa norte del Golfo San Matías (Pcia. de Río Negro, Argentina) detectaron la presencia de individuos inmaduros de otáridos en varias localidades arqueológicas. Dada las similitudes existentes a nivel osteológico (postcraneal) entre las dos especies identificadas en el registro de la costa patagónica (Otaria flavescens y Arctocephalus australis), desarrollamos un estudio osteométrico sobre materiales modernos (con edad y sexo conocido), con el objeto de distinguirlas. Los datos generados fueron contrastados con mediciones obtenidas sobre elementos inmaduros arqueológicos de la costa norpatagónica argentina. Los resultados evidencian que ésta metodología tiene un elevado potencial para discriminar las especies y, de esta forma, discutir cuestiones vinculadas con las estrategias de caza de lobos marinos en el pasado.

Palabras clave: Osteometría, Otaria flavescens, Arctocephalus australis, Zooarqueología. Norpatagonia.

\section{ABSTRACT}

Archaeofaunal analyses carried out in the northern coast of the San Matías Gulf (Río Negro, Argentina) have detected the presence of immature individuals of otariids in several sites. Due to the postcranial osteological similarities between the two recognized species (Otaria flavescens and Arctocephalus australis), we developed an osteometrical study on modern materials with known age and sex, in order to distinguish between them. The generated database was compared with measurements obtained on immature elements from northern Patagonia archaeological coastal sites (Argentina). The results show that this method has a high potential for discriminate species and discussing strategies of sea lions hunting in the past.

Key words: Osteometry, Otaria flavescens, Arctocephalus australis, Zooarchaeology, Northern Patagonia

i CONICET- INCUAPA, UNCPBA. Av del Valle 5737 (B7400JWI) Olavarría, Pcia de Bs.As. Argentina. Correo-e: fborella@ soc.unicen.edu.ar

ii CONICET- IMHICIHU. Saavedra 15, piso 5 (ACA1083), Buenos Aires. Argentina. Correo-e: lorenalheureux@yahoo. com.ar

Recibido: 03-12-2012 Revisado: 06-05-2013 Aceptado: 20-12-2013 


\section{INTRODUCCIÓNY OBJETIVOS}

A fines de los años $80^{\prime}$ se plantearon las dificultades existentes en el reconocimiento de los mamíferos marinos recuperados en sitios canoeros de Patagonia meridional. En vistas de avanzar sobre la discusión de la explotación de ambientes marinos y sus recursos, Legoupil (1989-90) propuso utilizar claves morfológicas en el cráneo y la mandíbula para la identificación de las dos especies de otáridos más frecuentes en el registro arqueofaunístico: el lobo fino (Arctocephalus australis) y el lobo común (Otaria flavescens).

Sin embargo, en muchos conjuntos zooarqueológicos, como en los de sitios de la costa atlántica de Patagonia continental, predominan los elementos postcraneales de estos animales. Por otra parte, dada la fusión tardía de los elementos del esqueleto postcraneal en ambas especies (véase cronograma de fusión en Borella et al. 2013a)sumado al marcado dimorfismo sexual que presentan ambas especies, complejiza aún más el panorama identificatorio, no pudiendo distinguirse el solapamiento específico y sexual de individuos nofusionados (e.g. Legoupil 1989-90:107). Esto condujo a que en muchos casos el estudio de estos taxa resultara elusivo, apareciendo mencionada la presencia de restos de ambas especies sin una cuantificación pormenorizada que permitiera discutir estrategias de explotación de estos animales en el pasado.

Este trabajo se propone explorar las posibilidades que tienen los estudios osteométricos para determinar especies de otáridos a partir de los restos postcraneales inmaduros (no-fusionados) representados en los conjuntos arqueofaunísticos. Para ello, se expone brevemente la metodología desarrollada y su aplicación arqueológica sobre una muestra ósea procedente de diferentes localidades litorales norpatagónicas (Argentina).

\section{ÁREA DE ESTUDIOSY PROBLEMA DE INVESTIGACIÓN}

Las investigaciones arqueológicas en concheros de mitílidos emplazados en la costa norte del Golfo San Matías -GSM- (Pcia. de Río Negro, Argentina) detectaron la presencia de restos de otáridos inmaduros -presumiblemente crías y subadultos-. Estos aparecieron asociados principalmente a restos de peces, aves y algunos vertebrados terrestres (Borella 2006, Borella et al. 20II). La identificación específica de Arctocephalus australis (Aa.) y de Otaria flavescens (Of.) pudo realizarse sólo en algunas localidades a partir del hallazgo de maxilares $y / o$ dientes, mientras que en otros se recuperaron únicamente restos del esqueleto postcraneal (Borella 2006). Por otra parte, los estudios de paleodietas humanas realizados en el área mostraron que los grupos de cazadoresrecolectores-pescadores tuvieron inicialmente una dieta predominantemente marina durante el Holoceno tardío inicial (del 3100 al 2200 AP.), para luego cambiar la señal isotópica hacia una dieta mixta durante el Holoceno tardío final (del I500 al 420 AP.) (Favier Dubois et al. 2009). En este marco, nos interesaba conocer el papel que tuvieron los mamíferos marinos en la subsistencia de estas poblaciones humanas $y$, para ello, desarrollamos un estudio osteométrico que permitiera identificar las especies representadas en el registro arqueológico, así como las clases de edad y sexo de los conjuntos óseos donde predominan individuos inmaduros.

\section{MATERIALESY MÉTODOS}

Para el estudio morfométrico se midieron todas las distancias lineales (L'Heureux y Borella 201I) posibles de ser relevadas en los huesos largos de un conjunto de lobos modernos. Esta muestra actual de referencia comprende individuos de ambas especies y fue colectada, en su totalidad, en la costa atlántica argentina (Figura la). La misma se encuentra constituida por 48 crías de hasta un año de Of. (ambos sexos igualmente representados) y ocho crías de Aa. (cuatro machos y cuatro hembras). La muestra de los subadultos es de uno a cuatro años de edad, y está representada por ocho individuos de Of. (tres machos y cinco hembras) y 13 individuos de Aa. (II machos y dos hembras), en suma 56 individuos de Of y 21 individuos de $A a$. La subrepresentación de Aa. en la colección de referencia, responde a que actualmente la presencia de apostaderos reproductivos de esta especie en el litoral patagónico argentino es sumamente escasa. Las muestras arqueológicas analizadas (N 8: cuatro tibias y cuatro húmeros), proceden de cinco sondeos realizados en diferentes localidades arqueológicas de la costa norte del GSM.A los efectos de este estudio 
fueron agrupadas en dos bloques temporales que se distinguen de acuerdo con las dietas humanas detectadas:A) bloque temporal temprano (ca. 31002200 AP.) donde los estudios isotópicos señalan una dieta marítima, se miden muestras de Bajada de los Pescadores, sondeo 2 (BaPesc); Barranca de los Concheros, sondeo I0 (BCH) y Faro San Matías, sondeo 2 (FSM), y B) bloque temporal tardío (ca. I500-420 AP.) con dietas mixtas, se miden muestras de Bajo de la Quinta-La Noria O (BQ-LNO) y Bahía Final, sondeo I0 (BF) (Figura Ib).

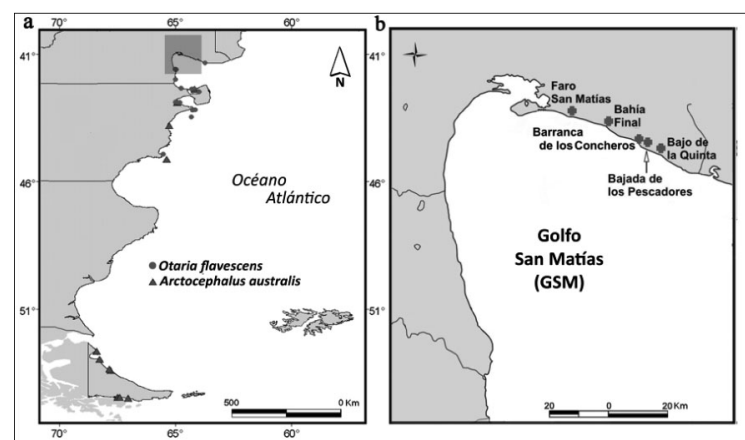

Figura 1: Procedencia de las muestras de lobos modernas (a) y localización de los sitios arqueológicos estudiados (b)

Figure 1: Provenance of modern sea lions samples (a) and archaeological sites analyzed $(b)$

Los patrones morfológicos generados a partir del análisis de la muestra moderna fueron comparados con los datos obtenidos de los elementos óseos recuperados en los concheros arqueológicos norpatagónicos.

En este trabajo,y por una cuestión de espacio, se presentan sólo los resultados osteométricos de dos elementos no fusionados: húmero (procedentes de BaPesc, FSM, BQ-LNO y BF) y tibia (procedentes de $\mathrm{BCH}, \mathrm{FSM}$ y dos de BQ-LNO). En estos huesos, tanto modernos como arqueológicos, se tomaron 18 variables métricas (véase descripción de las variables en L'Heureux y Borella 20 I I). Los datos obtenidos fueron analizados a partir de una estadística descriptiva exploratoria y de diferentes test estadísticos multivariados (Análisis de Componentes Principales -PCA- y Análisis Discriminante -AD-) utilizando los softwares PAST 2.13 y SYSTAT 12.0.

\section{RESULTADOS}

El componente I del PCA de húmeros y tibias explica más del $90 \%$ de la varianza. Los AD realizados para estos mismos huesos (Wilks' lambda 0,087; $P<0,00$ y Wilks' lambda 0,122; $P<0,00$ respectivamente), asignan correctamente entre el 75 y $95 \%$ de los casos según su sexo y especie. En el scatterplot del PCA de húmeros y tibias se observa que los casos arqueológicos caen dentro de la distribución de Aa., pero el sexo no resulta fácilmente distinguible (Figura 2 a y b). El mismo resultado se observa en la salida gráfica de los AD (Figura 3 a y b).
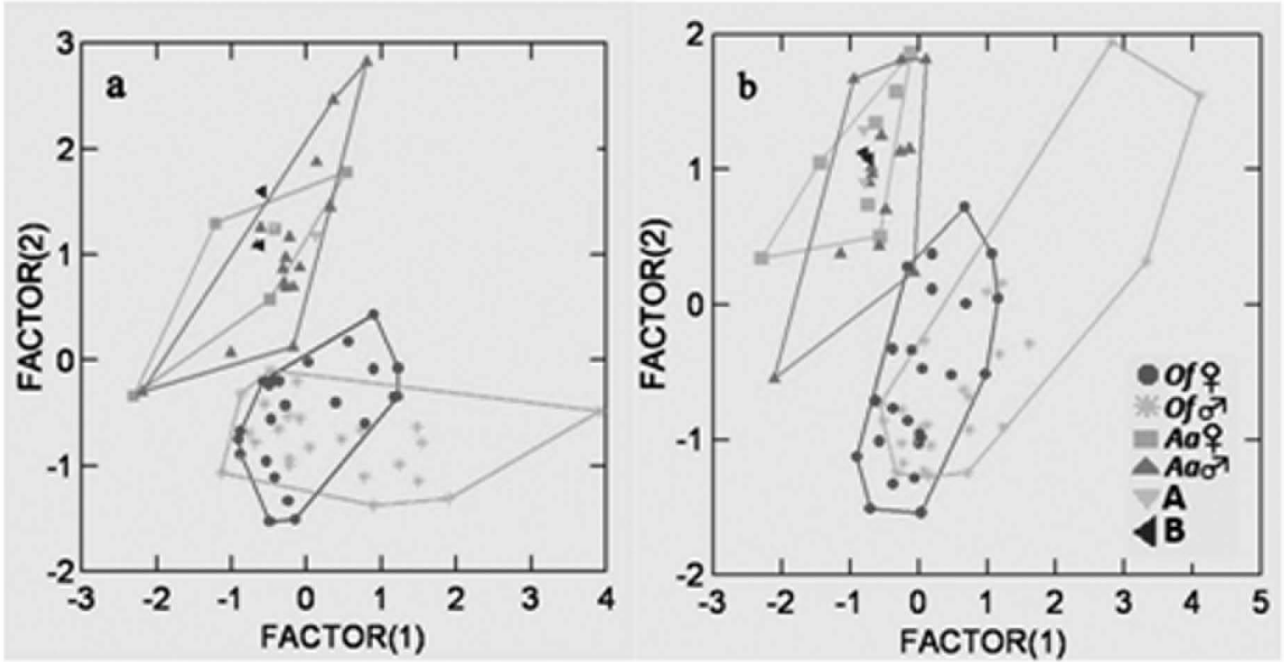

Figura 2: Scatterplot PCA húmeros (a) y tibias (b) modernas y arqueológicas

Figure 2: PCA scatter plot of modern and archaeological humeri (a) and tibias (b) 
Es importante mencionar que en las muestras modernas de cachorros el dimorfismo sexual sólo se registró en las tibias de Of., no así en el los húmeros de ambas especies. Esto pudo deberse a que el $72,23 \%$ de esta muestra tiene menos de un año, influyendo en la baja discriminación de éste parámetro en los huesos arqueológicos.
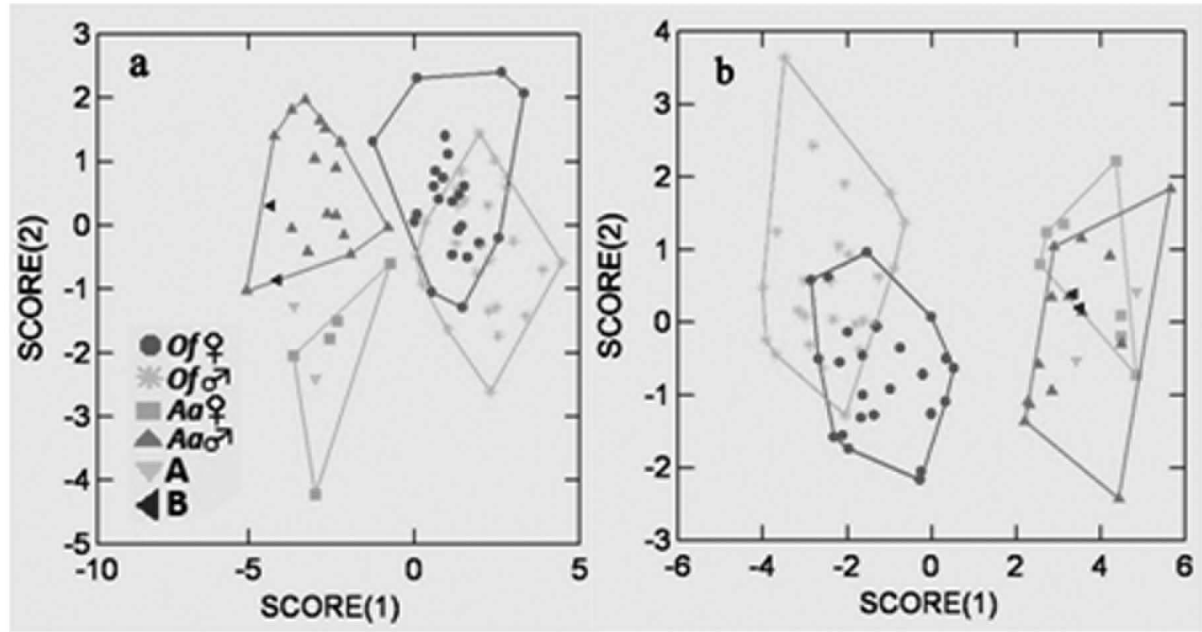

Figura 3: Scatterplot AD húmeros (a) y tibias (b) modernas y arqueológica

Figure 3: DA scatter plot of modern and archaeological humeri (a) and tibias (b)

Si bien la muestra de referencia actual para $A a$. es aún pequeña, observamos que es igualmente posible diferenciar ambas especies al menos en los dos huesos seleccionados para este estudio y dentro del rango de edad trabajado (de uno a cuatro años).

Asimismo, la variación morfológica intraespecífica que eventualmente podría existir en la muestra actual de $\mathrm{Aa}$. -incluye algunos individuos de Tierra del Fuego que podrían ser ligeramente más grandes que los del norte de Patagonia (véase discusión de tamaños por sexo en San Román 2007)-, tampoco pareciera haber afectado los resultados, permitiendo igualmente discriminar las dos especies, al menos en el rango etario considerado.

\section{CONCLUSIONES}

Uno de los principales limitantes en el análisis zooarqueológico de otáridos en Sudamérica ha sido la identificación taxonómica, particularmente a nivel postcráneo. En relación a ello y a modo de complementar sus estudios, Legoupil (1989-90:1056) había propuesto la búsqueda de claves en huesos largos, objetivo que estaba pendiente en la agenda zooarqueológica latinoamericana y que actualmente estamos desarrollando (Borella et al. 20/3b). Hemos presentado resultados que demuestran que la osteometría es una metodología promisoria para discriminar especies de otáridos, detectando diferencias sutiles de tamaños en huesos largos nofusionados, permitiendo estimar la proporción en que ambas especies fueron capturadas y explorar estrategias de explotación en lobos marinos a lo largo del tiempo.

De acuerdo con estos primeros estudios, los huesos arqueológicos inmaduros (crías y subadultos) hallados en las costa rionegrina corresponden mayoritariamente a Aa., mientras que Of. aparece representado por individuos de clases etarias mayores, reconocidos por fragmentos óseos de grandes dimensiones fusionados. Aunque estos estudios son preliminares (sólo presentamos el análisis de dos huesos), podría plantearse que se observa el predominio de Aa. en el registro arqueofaunístico rionegrino, lo que podría estar señalando la preferencia de esta especie como presa de caza. De confirmarse esta tendencia, se evidenciaría su mayor abundancia en el pasado respecto al presente. Siendo actualmente Of. la 
especie más abundante y la mejor representada en la costa atlántica argentina, es posible inferir notables cambios en la distribución de los otáridos respecto al pasado.

Hoy en día la distribución de Aa. está acotada a unos pocos enclaves en la costa patagónica, mientras que para el litoral del GSM se registró recientemente un pequeño apostadero reproductivo en el área Islote Lobos (Svendsen et al. 2013). En tal sentido, los resultados obtenidos proveen información paleoecológica más allá de los alcances netamente arqueológicos.

Agradecimientos: Al Dr. Kike Crespo y a los miembros de su equipo (Dra. F. Grandi; Lics. D. Vales y N. García del LAMAMA, CENPATCONICET) quienes determinaron sexo y edad de todas las muestras de referencia utilizadas (Colección Científica del LAMAMA-CONICET). A Natalie Goodall por permitirnos medir individuos de Aa. (Museo Acatushún, Tierra del Fuego). Las investigaciones en el GSM son financiadas por sucesivos Proyectos de Investigación otorgados por el CONICET (PIPI I 2-20080 I-00756 y PIPI I 220 I I 0 I-00589) y la Agencia FONCYT (PICT38264), Argentina. A la Agencia Río Negro Cultura y a la Secretaria de Ambiente y Desarrollo Sustentable de la Pcia. de Río Negro por otorgar los permisos para desarrollar las investigaciones arqueológicas en la región.A la Unidad Ejecutora INCUAPA-CONICET (UNCPBA). A dos evaluadores anónimos cuyas sugerencias mejoraron la comprensión del trabajo.

\section{BIBLIOGRAFÍA}

Borella, F. 2006. "¿Dónde están los lobos en la costa atlántica de Nordpatagonia? Explorando vías para resolver el registro arqueofaunístico". Werken 9: 97-II4.

Borella, F., F.L. Scartascini y H.A. Marani $201 \mathrm{l}$. "Explorando la subsistencia humana a partir de la evidencia faunística en la costa rionegrina". En Arqueología de Pescadores y Marisqueadores en Nordpatagonia. Descifrando un registro de más de 6000 años, compilado por F. Borella y M. Cardillo, Pp. 87-I I0. Dunken, Buenos Aires.

Borella, F., M.F. Grandi, D. Vales, R.N.P. Goodall y E.A. Crespo. 20I3a. "Esquema preliminar de fusión epifisaria en huesos de lobos marinos (Arctocephalus australis y Otaria flavescens), su contribución en los análisis zooarqueológicos". En Tendencias teórico-metodológicas y casos de estudio en la arqueología de Patagonia, compilado por A. F. Zangrando y R. Barberena, pp.395l. Editorial Altuna, Buenos Aires.

Borella, F., D. Vales, M. F. Grandi, N.A. García y E.A. Crespo. 20l3b. "Rasgos morfológicos en huesos del postcráneo para la identificación de otáridos en el registro zooarqueológico". En Libro de resúmenes del III Congreso nacional de Zooarqueología, PP. 34. UBA- FFyL, Tilcara, Jujuy.

Favier-Dubois, C.M., F. Borella y R. Tykot. 2009. "Tendencias en el uso humano del espacio y los recursos en el litoral rionegrino durante el Holoceno medio y tardío". En Arqueología de Patagonia:una mirada desde el último confin, compilado por M. Salemme F. Santiago, M. Álvarez, E. Piana, M.Vázquez y M.E. Mansur, pp. 985-998. Editorial Utopías, Ushuaia.

L'Heureux, G.L. y F. Borella. 20I I. Guía Osteométrica para el Estudio de Elementos Óseos de Otaria flavescens. UNCPBA, Tandil.

Legoupil, D. 1989-1990. "La identificación de los mamíferos marinos en los sitios canoeros de Patagonia; problema y constataciones". Anales del Instituto de la Patagonia 19: I0I-II3.

San Román, M. 2007. "La explotación de mamíferos marinos en el sitio de Bahía Buena: economía de canoeros tempranos de Patagonia (Estrecho de Magallanes, Chile)". En Arqueología de Fuego-Patagonia. Levantando piedras, desenterrando huesos...y develando arcanos, editado por F. Morello, M. Martinic, A. Prieto y G. Bahamonde , pp. 295-3 I0. Ediciones CEQUA, Punta Arenas.

Svendsen, G.M, S.L. Dans, R.A. González, M.A. Romero y E.A. Crespo. 2013."Occurence of South American fur seals Arctocephalus australis (Zimmermann, 1783) in San Matías Gulf, Patagonia, Argentina". Latin American Journal of Aquatic Resources 4I(3):576-583. 